\title{
Telemedicine in Rheumatology
}

\author{
Haydar GÖK \\ Editor
}

Telemedicine can be defined as the use of information and telecommunication technology to provide patients with healthcare at a distance. Healthcare professionals are increasingly faced with the daunting task of caring for a rapidly aging and ever expanding population. Hence, the main aims of telemedicine are to reduce pressure on secondary or tertiary healthcare services and to provide clinical care at a distance. There are now many applications for telemedicine such as telecare, distant interpretation of digitally stored radiographs, magnetic rezonance scans or photographs of skin lesions, teleconsultations, referral services, remote monitoring of patients with chronic conditions, online continuing medical education (CME), and online provision of medical and health information to patients. It is especially beneficial for those in rural areas because it reduces the need to travel for medical care along with the related costs. There is a critical shortage of rheumatology services in Turkey, and the impact is felt more in rural and eastern regions of the country. Even though telemedicine in Turkey began comparatively late compared with developed countries, there has been considerable recent development.

Current research shows evidence of the effectiveness and acceptability of telemedicine in the rheumatology field. Davis et al. ${ }^{[1]}$ showed that teleconsultations in rheumatology were both feasible and acceptable. They found that patients were satisfied with this method, and $84 \%$ felt that the care received was as good as an "in person" visit. Jong et al. ${ }^{[2]}$ investigated the utility of telehealth for providing rheumatology services to rural and northern communities in Canada and found that videoconferencing was preferable to clinic visitation and email in remote areas. Leggett et al. ${ }^{[3]}$ examined the diagnostic accuracy and acceptability of telemedicine in the field of rheumatology and found that the televisual consultations were highly accurate (97\%) and acceptable to patients, general practitioners, and specialists. Roberts et al. ${ }^{[4]}$ reported on the telerheumatology experience in Australia and emphasized the need for face-to-face consultations alongside teleconsultations to ensure that deficiencies in telehealth can be addressed in specific situations.

Many physicians remain skeptical and think that telemedicine should have a limited role in diagnosis since rheumatology is a practice that is highly based on physical examination. They suggest that a more suitable role for telemedicine could be in the delivery of ongoing care to patients that have been definitively diagnosed by a rheumatologist. In a systematic review supporting this idea, the MartinKhan et al. ${ }^{[5]}$ concluded that in studies in which a physical examination was a part of the diagnostic process, the results were reliable. However, the authors recommended that greater care be taken during this process (e.g., good equipment, onsite support, additional camera angles). This demonstrates that physicians still need to be reassured that the technology used in telemedicine is adequate and reliable.

Based on recent advances in videoconferencing and information technology, one can predict that telemedicine will be used widely in rheumatology practice in the future and that it will have a substantial impact on many aspects of healthcare as more physicians embrace this technology.

\section{Declaration of conflicting interests}

The author declared no conflicts of interest with respect to the authorship and/or publication of this article.

\section{Funding}

The author received no financial support for the research and/or authorship of this article.

\section{REFERENCES}

1. Davis P, Howard R, Brockway P. An evaluation of telehealth in the provision of rheumatologic consults to a remote area. J Rheumatol 2001;28:1910-3.

2. Jong M, Kraishi M. A comparative study on the utility of telehealth in the provision of rheumatology services to rural and northern communities. Int J Circumpolar Health 2004;63:415-21.

3. Leggett P, Graham L, Steele K, Gilliland A, Stevenson M, O'Reilly $\mathrm{D}$, et al. Telerheumatology--diagnostic accuracy and acceptability to patient, specialist, and general practitioner. Br J Gen Pract 2001;51:746-8.

4. Roberts LJ, Lamont EG, Lim I, Sabesan S, Barrett C. Telerheumatology: an idea whose time has come. Intern Med J 2012;42:1072-8. doi: 10.1111/j.1445-5994.2012.02931.x.

5. Martin-Khan M, Wootton R, Whited J, Gray LC. A systematic review of studies concerning observer agreement during medical specialist diagnosis using videoconferencing. J Telemed Telecare 2011;17:350-7. doi: 10.1258/jtt.2011.101113. 